\title{
Article \\ Significance of Bernoulli Integral Terms for the Solar Wind Protons at 1 au
}

\author{
Georgios Nicolaou ${ }^{1, *(\mathbb{D}}$, George Livadiotis ${ }^{1}$ and Mihir I. Desai ${ }^{1,2}$ \\ 1 Southwest Research Institute, San Antonio, TX 78238, USA; george.livadiotis@swri.org (G.L.); \\ mihir.desai@swri.org (M.I.D.) \\ 2 Department of Physics and Astronomy, University of Texas at San Antonio, San Antonio, TX 78249, USA \\ * Correspondence: georgios.nicolaou@swri.org
}

Citation: Nicolaou, G.; Livadiotis, G.; Desai, M.I. Significance of Bernoulli Integral Terms for the Solar Wind Protons at 1 au. Appl. Sci. 2021, 11, 4643. https://doi.org/10.3390/ app11104643

Academic Editor: Jochen Schein

Received: 20 March 2021

Accepted: 14 May 2021

Published: 19 May 2021

Publisher's Note: MDPI stays neutral with regard to jurisdictional claims in published maps and institutional affiliations.

Copyright: (c) 2021 by the authors. Licensee MDPI, Basel, Switzerland. This article is an open access article distributed under the terms and conditions of the Creative Commons Attribution (CC BY) license (https:// creativecommons.org/licenses/by/ $4.0 /)$.

\begin{abstract}
The Bernoulli integral describes the energy conservation of a fluid along specific streamlines. The integral is the sum of individual terms that contain the plasma density, speed, temperature, and magnetic field. Typical solar wind analyses use the fluctuations of the Bernoulli integral as a criterion to identify different plasma streamlines from single spacecraft observations. However, the accurate calculation of the Bernoulli integral requires accurately determining the plasma polytropic index from the analysis of density and temperature observations. To avoid this complexity, we can simplify the calculations by keeping only the dominant terms of the integral. Here, we analyze proton plasma and magnetic field observations obtained by the Wind spacecraft at $1 \mathrm{au}$, during 1995 . We calculate the Bernoulli integral terms and quantify their significance by comparing them with each other. We discuss potential simplifications of the calculations in the context of determining solar wind proton thermodynamics using single spacecraft observations.
\end{abstract}

Keywords: solar wind; space plasmas; plasma dynamics

\section{Introduction}

The solar wind is a very dynamic system, with plasma and electromagnetic field fluctuations occurring in a wide range of scales (e.g., [1], and references therein). Plasma and field measurements should be analyzed properly in order to understand the mechanisms that govern solar wind and space plasmas in general. For instance, knowing the velocity distribution functions of the plasma species makes it possible to derive the plasma bulk parameters, which are crucial in investigating mechanisms that involve momentum, heat, and energy transfer.

Certain dynamical processes need to be investigated along specific streamlines. If plasma observations are obtained in different streamlines, plasmas with entirely different properties may end up being analyzed, regardless of the mechanisms acting on them. Different streamlines in the solar wind may contain plasmas that originated in vastly different structures of the Sun. Therefore, if we do not analyze specific streamlines separately, the observed plasma variations may reflect the different plasma sources and not the effects of interesting dynamical processes.

For example, empirical determination of the plasma polytropic index from the plasma density and temperature fluctuations has meaning only for observations obtained within the same plasma streamline (e.g., [2,3]). The Bernoulli integral, which is expressed in terms of energy per mass, is conserved along specific streamlines. Therefore, typical analyses can identify plasma streamlines by detecting time intervals for which the standard deviation of the Bernoulli integral is below a predefined threshold value (e.g., [4-9]).

The Bernoulli integral is given by

$$
\frac{1}{2} v^{2}+\frac{\gamma}{\gamma-1} \frac{P}{\rho}+\frac{B^{2}}{2 \rho \mu_{0}}=C \text { for } \gamma \neq 1,
$$


and

$$
\frac{1}{2} v^{2}+\ln \rho \frac{P}{\rho}+\frac{B^{2}}{2 \rho \mu_{0}}=C \text { for } \gamma \neq 1,
$$

where $v$ is the plasma bulk speed, $P$ is the thermal pressure, $\rho$ is the mass density, $B$ is the magnetic field, $\gamma$ is the polytropic index, $\mu_{0}$ is the magnetic permeability constant, and $C$ is a constant characteristic for a streamline. The first term of the integral is the dynamic pressure over $\rho$. The second term includes the thermal pressure normalized to $\rho$, while the third term is the magnetic field pressure over $\rho$. Note that the fraction $1 / 2$ in the third term, comes from averaging over all the possible angles between $\boldsymbol{B}$ and $\boldsymbol{v}$ vectors (e.g., $[10,11])$. The Bernoulli integral, in terms of the bulk flow speed $v$, thermal speed $v_{\text {th }}$, and Alfvén speed $v_{\mathrm{A}}$, is:

$$
\frac{1}{2} v^{2}+\frac{1}{2} v_{\mathrm{th}}^{2}\left(\frac{\gamma}{\gamma-1}\right)+\frac{1}{2} v_{A}^{2}=C \text { for } \gamma \neq 1
$$

and

$$
\frac{1}{2} v^{2}+\frac{1}{2} v_{\text {th }}^{2} \ln \rho+\frac{1}{2} v_{A}^{2}=C \text { for } \gamma \neq 1
$$

In general, the first (dynamic) term is expected to be the dominant term in solar wind protons at $\sim 1 \mathrm{au}$, where the bulk flow speed is typically larger than the thermal and the Alfvén speed. However, the second term may become significant as $\gamma \rightarrow 1$.

In this study, we investigate the significance of the Bernoulli integral terms in solar wind proton plasma. We analyze proton observations obtained at 1 au in order to quantify the contribution of each term and examine the accuracy of specific simplifications. We firstly investigate the integral terms for theoretical values of $\gamma$ in order to cover a wide range of possible values. We then investigate the integral terms by estimating the actual $\gamma$ of solar wind protons from the density and temperature fluctuations. The specific investigation reveals the occurrence of solar wind proton intervals in which the thermal term dominates over the other two terms. Based on this result we decide whether or not is appropriate to simplify the calculations in future applications. Here, we analyze an annual interval that contains observations of both slow and fast solar wind plasma (see [7,12]). In Section 2, we describe our data set, while in Section 3, we present the results of our analysis. In Section 4, we examine a continuous expression for the Bernoulli integral, and in Section 5, we discuss our findings. Finally, Section 6 summarizes our conclusions.

\section{Data}

We use the Wind spacecraft observations obtained at 1 au during 1995. We analyze the high time-resolution ( $\sim 92 \mathrm{~s}$ ) proton parameters and their 1-sigma uncertainties derived from non-linear fittings of bi-Maxwellian distributions to the observed distribution functions [13]. The quality of each fitting is quantified by the chi-squared parameter $\chi^{2}$. Here, we use the proton plasma density $n$, bulk speed $v$, thermal speed $v_{\text {th }}$, the 1-sigma error of the plasma density $\sigma_{n}$, and thermal speed $\sigma_{v}$,th . We exclude the parameters that are derived with $x^{2}>5$ and all data points with relative density and temperature uncertainty $\frac{\sigma_{n}}{n}>0.3$ and $\frac{\sigma_{T}}{T}>0.3$, respectively. Finally, we use the magnetic field $B$ observations averaged over the time resolution of the plasma data $(\sim 92 \mathrm{~s})$.

\section{Results}

\subsection{Bernoulli Integral}

Figure 1 a shows the terms $\frac{1}{2} v^{2}, \frac{1}{2} v_{\mathrm{th}}^{2}$, and $\frac{1}{2} v_{\mathrm{A}}^{2}$ within the analyzed data set as a function of time. The gray line shows the dynamic term $\frac{1}{2} v^{2}$, while the red and blue lines show the thermal $\frac{1}{2} v_{\mathrm{th}}^{2}$ and the magnetic $\frac{1}{2} v_{\mathrm{A}}^{2}$ terms, respectively. The dynamic term is almost always larger than the other two terms. Figure $1 \mathrm{~b}$ shows histograms of the logarithms of the integral terms as calculated for the same time interval. The dynamic term peaks at $\sim 4 \times 10^{10} \mathrm{~m}^{2} \mathrm{~s}^{-2}$. The thermal term peaks at $\sim 3 \times 10^{8} \mathrm{~m}^{2} \mathrm{~s}^{-2}$ and the magnetic term at $6 \times 10^{8} \mathrm{~m}^{2} \mathrm{~s}^{-2}$. The magnetic term ranges below and above the thermal, and slightly 
overlaps the dynamic term. The dynamic and thermal terms have no common range of values.
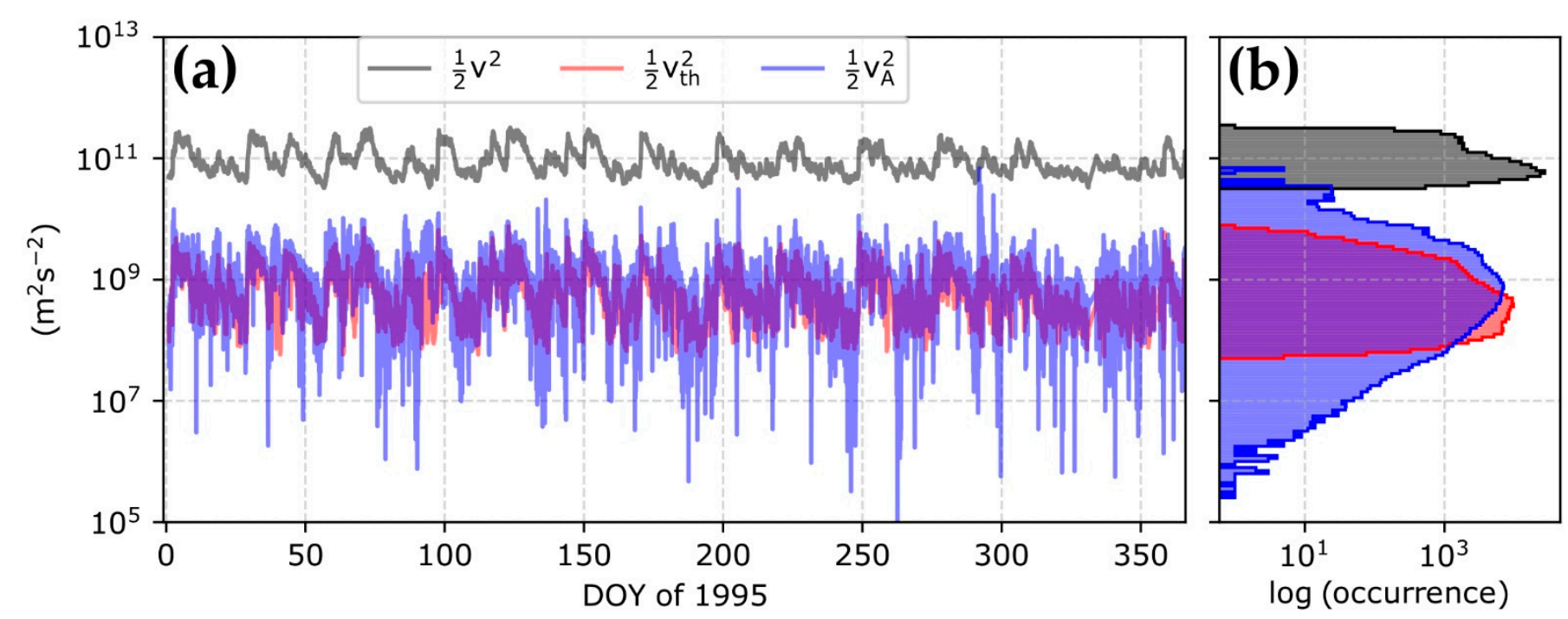

Figure 1. (a) The (gray) $\frac{1}{2} v^{2}$, (red) $\frac{1}{2} v_{\mathrm{th}}^{2}$, and (blue) $\frac{1}{2} v_{\mathrm{A}}^{2}$ terms calculated for solar wind protons at $\sim 1$ au during 1995 , and (b) the corresponding histograms of each term. The dynamic term $\frac{1}{2} v^{2}$, is almost always larger than the other two.

According to Equation (3), the second term of the non-isothermal Bernoulli integral is the thermal $\frac{1}{2} v_{\text {th }}^{2}$ multiplied by $\frac{\gamma}{\gamma-1}$. Thus, the significance of the second term varies with the polytropic index $\gamma$. Figure 2a plots the time series of the Bernoulli integral in the solar wind protons, assuming five different values for $\gamma$. We specifically assume isobaric protons $(\gamma=0)$, protons with $\gamma=1 / 2$, protons with $\gamma=3 / 2$, adiabatic protons with three kinetic degrees of freedom $\left(\gamma=c_{\mathrm{p}} / c_{\mathrm{v}}=1.67\right)$, and protons with a nearly isochoric behavior $(\gamma \rightarrow \infty)$. Figure $2 \mathrm{~b}$ focuses on a subinterval ranging from day of year (DOY) 305 to DOY 310. In Figure 2c, we show the histograms of the Bernoulli integral as calculated for all of 1995 for the different $\gamma$ values. Our results shows that there are no apparent differences in the calculated integral, regardless of the $\gamma$ values.

The second term of the Bernoulli integral may become the most significant term as $\gamma \rightarrow 1$ (nearly isothermal plasma). In Figure 3 a, we show $\frac{\gamma}{\gamma-1}$ as a function of $\gamma$. The grey shadow indicates the range $5 / 6<\gamma<5 / 4$ for which the absolute value of $\frac{\gamma}{\gamma-1}$ exceeds 5 . Figure $3 \mathrm{~b}$ shows the histograms of the dynamic term, and the thermal term multiplied by 5 . The two histograms overlap within the small range from $3.3 \times 10^{10}$ to $3.7 \times 10^{10} \mathrm{~m}^{2} \mathrm{~s}^{-2}$. This result means that in the data we analyze, the second term of the Bernoulli integral may become comparable to the dynamic term for $5 / 6<\gamma<5 / 4$. 

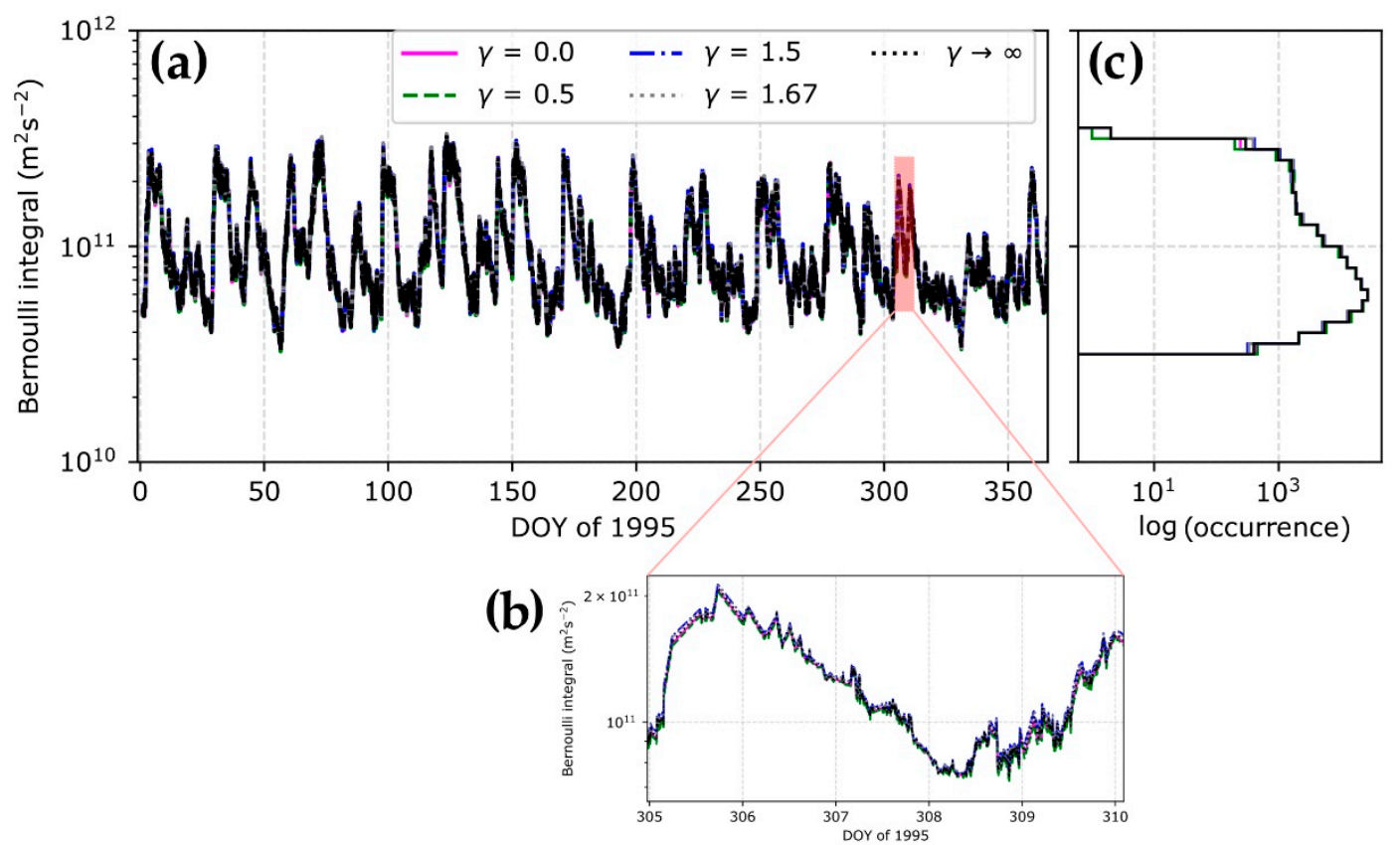

Figure 2. (a) Time series of the Bernoulli integral for different polytropic index values; (solid magenta) $\gamma=0$, (dashed green) $\gamma=1 / 2$, (dashed dotted blue) $\gamma=3 / 2$, (dotted grey) $\gamma=5 / 3$, and (dotted black) $\gamma \rightarrow \infty$. (b) The Bernoulli integral for the considered $\gamma$ values in a short sub-interval from day 305 to day 310 of 1995. (c) Histograms of the Bernoulli integral in 1995 for the different polytropic indices we assume here.
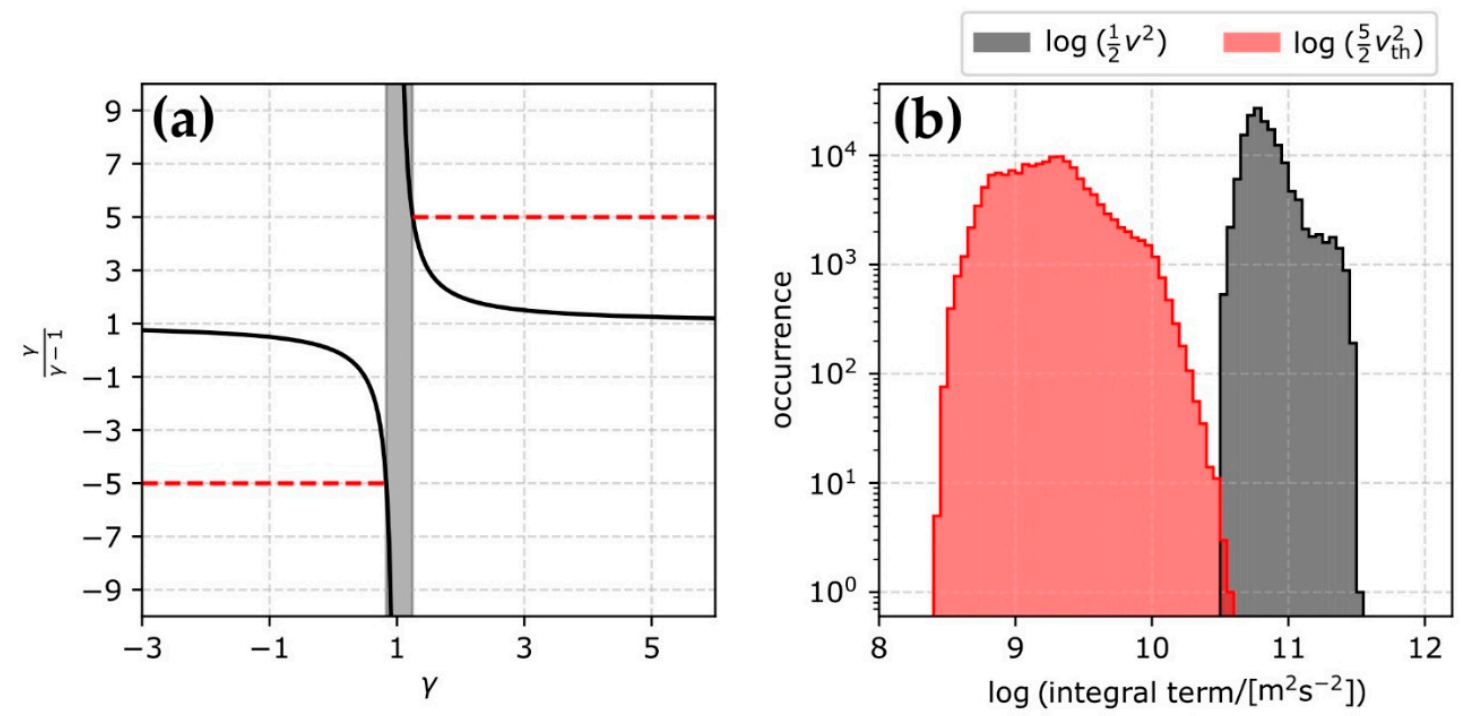

Figure 3. (a) The term $\frac{\gamma}{\gamma-1}$ as a function of $\gamma$. The grey ribbon indicates the range of $\gamma$ for which the absolute value of $\frac{\gamma}{\gamma-1}$ exceeds 5 (red dashed). (b) the histograms of (grey) the dynamic term and (red) the absolute value of the thermal term of the Bernoulli integral assuming plasma with $\frac{\gamma}{\gamma-1}=5$ (or -5 ).

\subsection{Variation of the Bernoulli Integral}

The Bernoulli integral is often used as a criterion to identify streamlines within the solar wind (e.g., [4-9]). Such analyses usually examine the standard deviation of the Bernoulli integral within selected time intervals. Ideally, if the plasma measurements within the selected data interval represent the same streamline, the standard deviation of the calculated Bernoulli integral should be zero. However, in data analyses we may classify observations with standard deviations smaller than a reasonable threshold value as streamlines. 
Here, we examine the standard deviation of the Bernoulli integral in short consecutive time intervals in 1995 . As in $[9,14,15]$, we analyze only time intervals that are shorter than $15 \mathrm{~min}$. The choice of short intervals reduces the possibility to analyze data-points from different streamlines. However, the intervals should be large enough to have enough data-points for accurate calculations (for more see $[9,14,15]$ ). We calculate the standard deviations over the absolute mean values of the Bernoulli integral $\frac{\sigma_{\text {Bernoulli }}}{\mid \text { Bernoulli } \mid}$ in each interval. We repeat the calculations, each time assuming a different polytropic index of the plasma protons. We specifically consider $\gamma=0, \gamma=5 / 6$, and $\gamma=5 / 4$. We also calculate the relative standard deviation of the dynamic term $\frac{\sigma_{\text {dynamic }}}{\text { dynamic }}$ within each interval. Figure 4 a shows the histograms of the calculated relative deviations, while Figure $4 \mathrm{~b}$ plots the histograms of the differences $\frac{\sigma_{\text {Bernoulli }}}{\mid \text { Bernoulli } \mid}-\frac{\sigma_{\text {dynamic }}}{\text { dynamic }}$, for each $\gamma$ value.

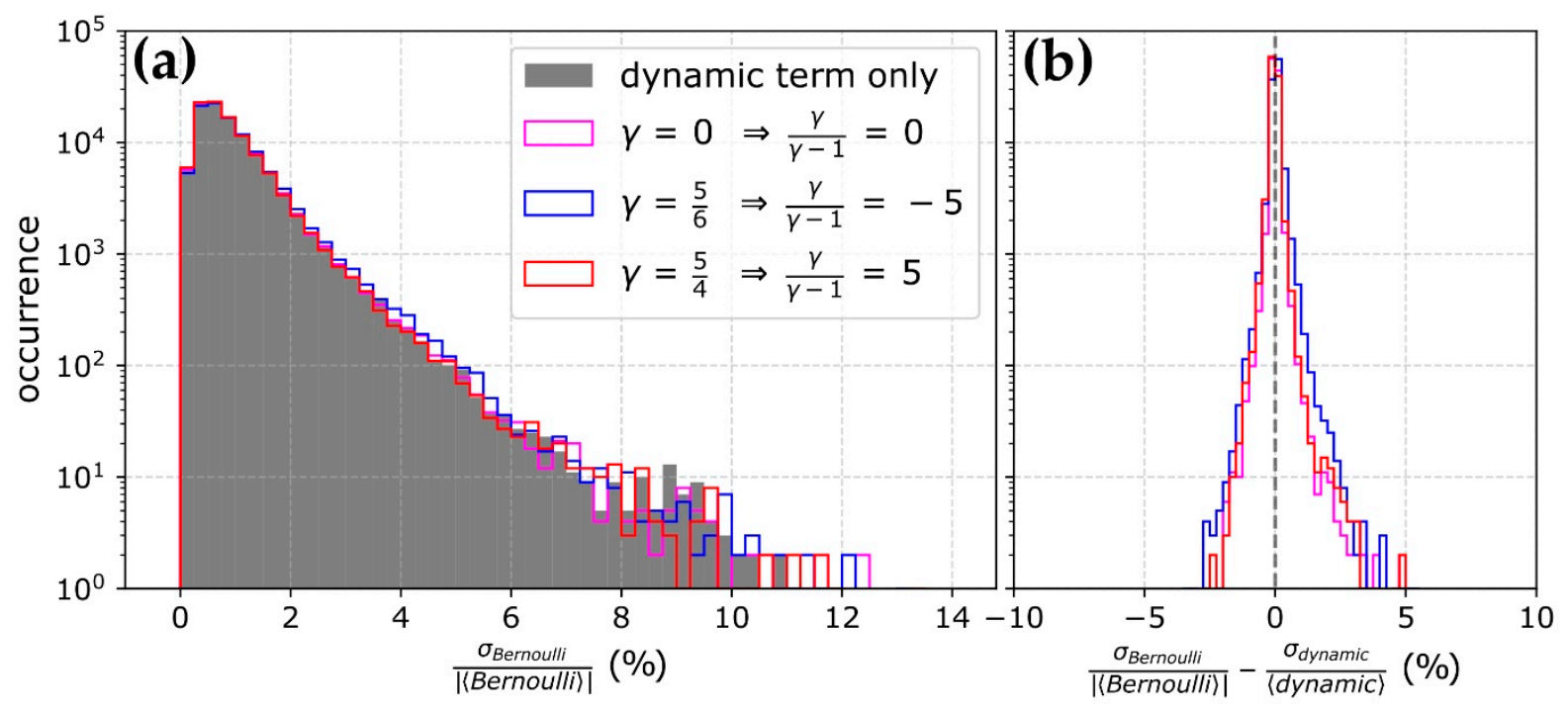

Figure 4. (a) Histograms of the relative standard deviations (standard deviations over the absolute mean values) of the Bernoulli integral within short time intervals observed in 1995. We calculate the Bernoulli integral assuming (magenta) $\gamma=0$, (blue) $\gamma=5 / 6$, and (red) $\gamma=5 / 4$. The grey histogram shows the relative deviation of the dynamic term $\frac{1}{2} v^{2}$. (b) histograms of the differences between the Bernoulli integral relative deviation and the relative deviation of the dynamic term within the selected intervals.

Our result indicates that the second and the third term of the integral do not significantly modify its relative deviation. For $\gamma=0$, the second term of the integral vanishes, since $\frac{\gamma}{\gamma-1}=0$. In this case, the relative standard deviation of the integral does not differ by more than $5 \%$ of the relative deviation of the dynamic term. This means that the magnetic term contributes no more than $\sim 5 \%$ to the relative deviations of the integral. For $\gamma=5 / 6$ $\left(\frac{\gamma}{\gamma-1}=-5\right)$, the second term becomes negative and reduces the mean value of the Bernoulli integral. This seems to have some minor effects on the relative standard deviations. We observe that the corresponding histogram of $\frac{\sigma_{\text {Bernoulli }}}{\mid \text { Bernoulli } \mid}-\frac{\sigma_{\text {dynamic }}}{\text { dynamic }}$ is slightly wider (by $\sim 1 \%$ ) than that for $\gamma=0$. Finally, for $\gamma=5 / 4$, we do not observe any apparent difference from the case with $\gamma=0$. According to this, we infer that even when $\gamma$ is very close to unity, we may completely ignore the second term of the Bernoulli integral with almost no effect on the accuracy of the calculated relative standard deviations.

\subsection{Propagation of Uncertainty}

The calculation of the second term in Equations (1) and (3) requires the determination of $\gamma$. For realistic plasma measurements with a certain level of uncertainty, the index $\gamma$ is derived with uncertainty $\delta_{\gamma}$. We use the error propagation formula in order to estimate the 
uncertainty of the function $F(\gamma) \equiv \frac{\gamma}{\gamma-1}$, which is part of the second term in the Bernoulli integral as

$$
\delta_{F}=\left|\frac{\vartheta F}{\vartheta \gamma}\right| \delta_{\gamma} \Rightarrow \delta_{F}=\frac{\delta_{\gamma}}{(\gamma-1)^{2}}
$$

The relative error $\frac{\delta_{F}}{F}$ is

$$
\frac{\delta_{F}}{F}=\frac{1}{(\gamma-1)} \frac{\delta_{\gamma}}{\gamma} .
$$

In Figure 5, we show the relative error $\frac{\delta_{F}}{F}$ as a function of $\gamma$ for different values of the relative error of the polytropic index $\frac{\delta_{\gamma}}{\gamma}$. The relative error $\frac{\delta_{F}}{F}$ increases rapidly as $\gamma \rightarrow 1$. The grey solid bar shows the range $5 / 6<\gamma<5 / 4$, for which the absolute value of $F$ is larger than 5 , and the histogram of the thermal term of the Bernoulli integral overlaps with the histogram of the dynamic term (see Figure 3). According to the diagrams in Figure 5, even for a small error in the calculated polytropic index such as $\frac{\delta_{\gamma}}{\gamma}=0.1$, the relative error of $F(\gamma)$ exceeds $\sim 50 \%$ for $\gamma$ values between $5 / 6$ and $5 / 4$.

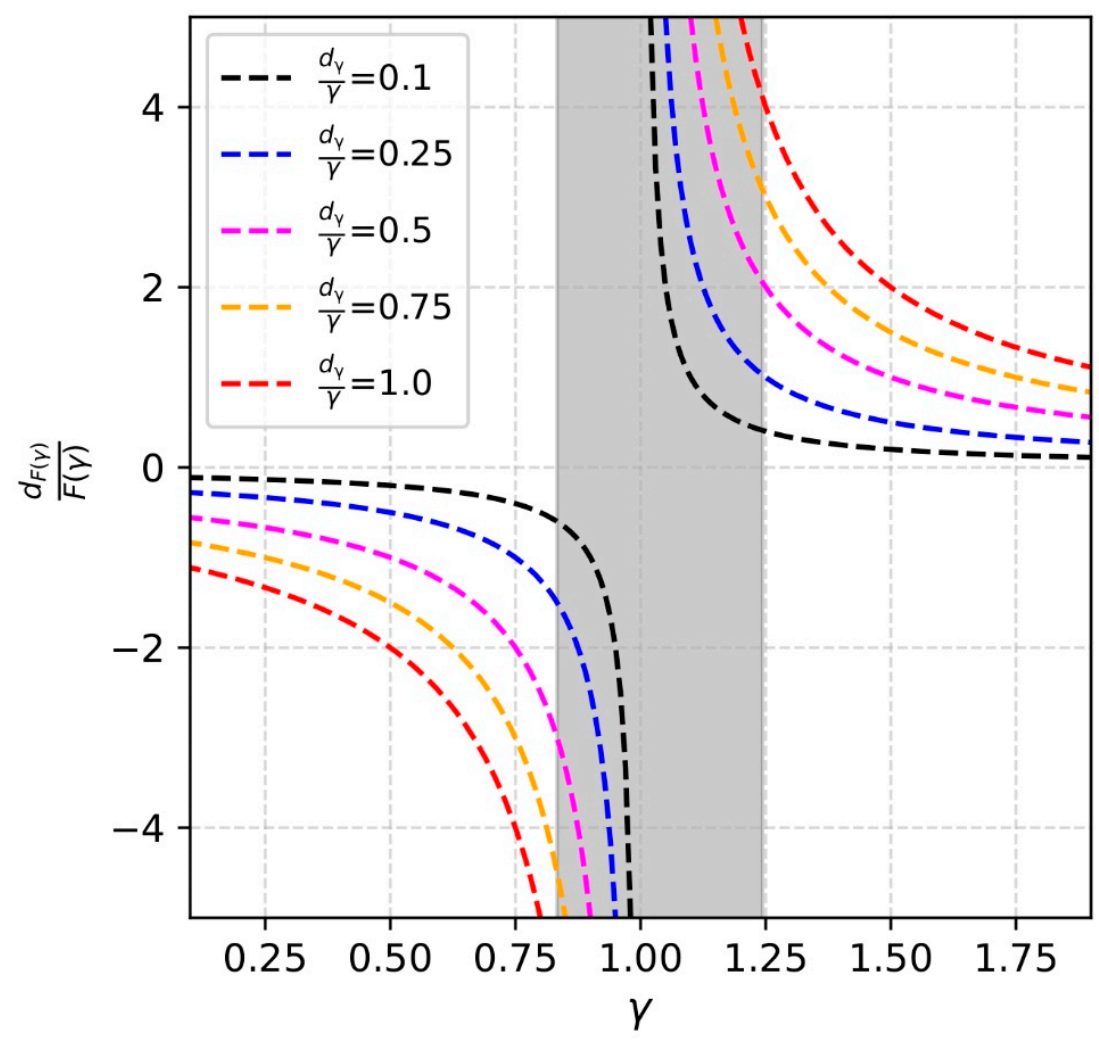

Figure 5. Relative error of the function $F(\gamma)=\frac{\gamma}{\gamma-1}$, as a function of $\gamma$ and for polytropic index uncertainties $\frac{\delta_{\gamma}}{\gamma}$.

Our analysis shows that the second term of the non-isothermal Bernoulli integral and its uncertainty may become significant for values $\gamma \rightarrow 1$. In typical analyses however, we may use the non-isothermal expression when $|\gamma-1|$ is bigger than a threshold value we choose arbitrarily. Then, the significance of the second term in the Bernoulli integral depends on the threshold we choose. For instance, if we choose to use the isothermal expression for $|\gamma-1|<0.3$, then according to Equations (2) and (4), the significance of the thermal term depends on the plasma density. It is then reasonable to ask: how do we choose the appropriate integral expression when $\gamma$ is close to unity? In the Section 4 we re-examine the formula of the Bernoulli integral. 


\section{The Complete Bernoulli Integral}

Livadiotis 2016 [16] shows step by step the derivation of the Bernoulli integral for compressible plasmas. They formulate the Bernoulli integral for any value of $\gamma$ as

$$
\frac{1}{2} v^{2}+\frac{1}{2} v_{\text {th }}^{2} \cdot \gamma \cdot \ln _{\gamma}\left(\frac{\rho}{\rho_{*}}\right)+\frac{1}{2} v_{A}^{2}=C,
$$

where $\rho$ is the plasma density and $\rho_{*}$ is an arbitrary scale, which could be the density value measured at any specific point within the streamline. Finally, $\ln _{\gamma}\left(\frac{\rho}{\rho_{*}}\right)$ is the q-deformed natural logarithm (e.g., [17-19]) defined as

$$
\ln _{\gamma}\left(\frac{\rho}{\rho_{*}}\right)=\frac{1-\left(\frac{\rho}{\rho_{*}}\right)^{1-\gamma}}{\gamma-1} .
$$

For $\gamma \rightarrow 1$, Equation (8) gives the natural logarithm $\ln \left(\frac{\rho}{\rho_{*}}\right)$. In Figure 6a, we plot the function $F^{\prime}(\gamma) \equiv \gamma \cdot \ln _{\gamma}\left(\frac{\rho}{\rho_{*}}\right)$ as a function of $\gamma$ and different values of $\frac{\rho}{\rho_{*}}$. The dotted horizontal lines indicate the values of the natural logarithm $\ln \left(\frac{\rho}{\rho_{*}}\right)$ that intersect $F^{\prime}$ at $\gamma=1$. The function $F^{\prime}(\gamma)$ is continuous at $\gamma=1$, and highly dependent on $\frac{\rho}{\rho_{*}}$ in general.

For a direct comparison with our results in Section 3, we calculate the integral as expressed in Equation (7) for $\gamma=5 / 6$, and $\gamma=5 / 4$ using the plasma proton observations in 1995. Figure $6 \mathrm{~b}$ shows the histograms of the dynamic and the second term of the integral. Although the spread of the second term is larger than when calculated using Equation (1) or (3), it does not overlap with the dynamic term.
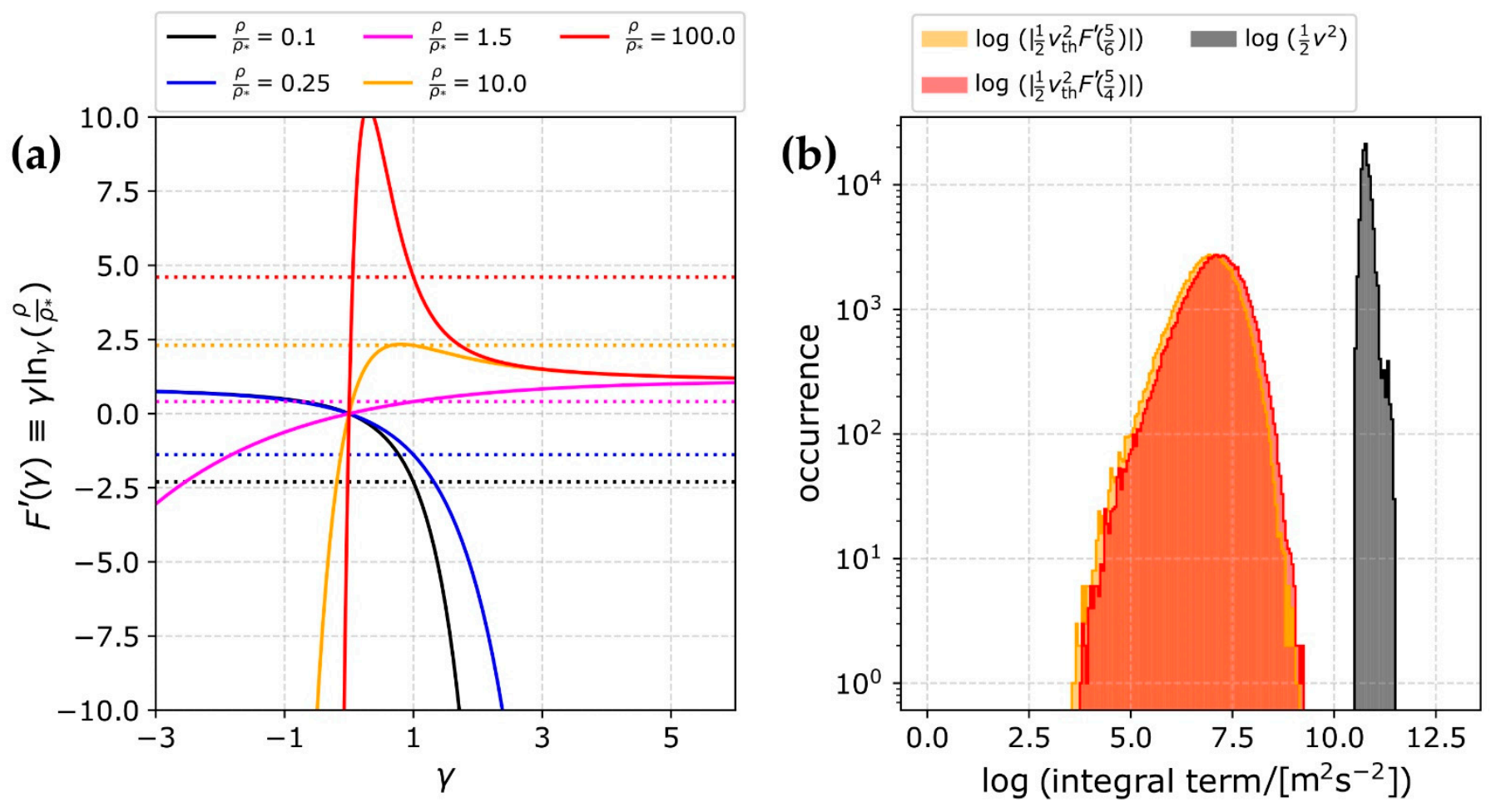

Figure 6. (a) The function $F^{\prime}(\gamma) \equiv \gamma \cdot \ln _{\gamma}\left(\frac{\rho}{\rho_{*}}\right)$, which multiplies the thermal term in the complete Bernoulli integral as a function of $\gamma$ and for several $\frac{\rho}{\rho_{*}}$ ratios. (b) Histograms of the (grey) dynamic term and the absolute value of the second term of Equation (7) as calculated from Wind observations, assuming (orange) $\gamma=5 / 6$, and (red) $\gamma=5 / 4$.

We also examine the complete Bernoulli integral for $\gamma$ determined from the density and temperature data within selected, short (<15 min) intervals. Similarly to $[9,20,21]$, 
we perform a weighted fitting of the polytropic equation to $\log (n)$ vs. $\log (T)$ data. The weighted fitting derives the polytropic index $\gamma$ and the 1-sigma error $\sigma_{\gamma}$. We apply a polytropic index uncertainty filter by excluding data points with $\sigma_{\gamma}$ larger than a specific threshold value. In Figure 7, we plot histograms of $\frac{1}{2} v^{2}$ and $\frac{1}{2} v_{\text {th }}^{2} F^{\prime}(\gamma)$ terms for different $\sigma_{\gamma}$ filters. When all the data points are taken into account, there is a small overlap of the two terms. However, the number of overlapping data points is negligible compared to the total data set. For all the other cases we present here, the second term of the integral does not become comparable with the dynamic term.

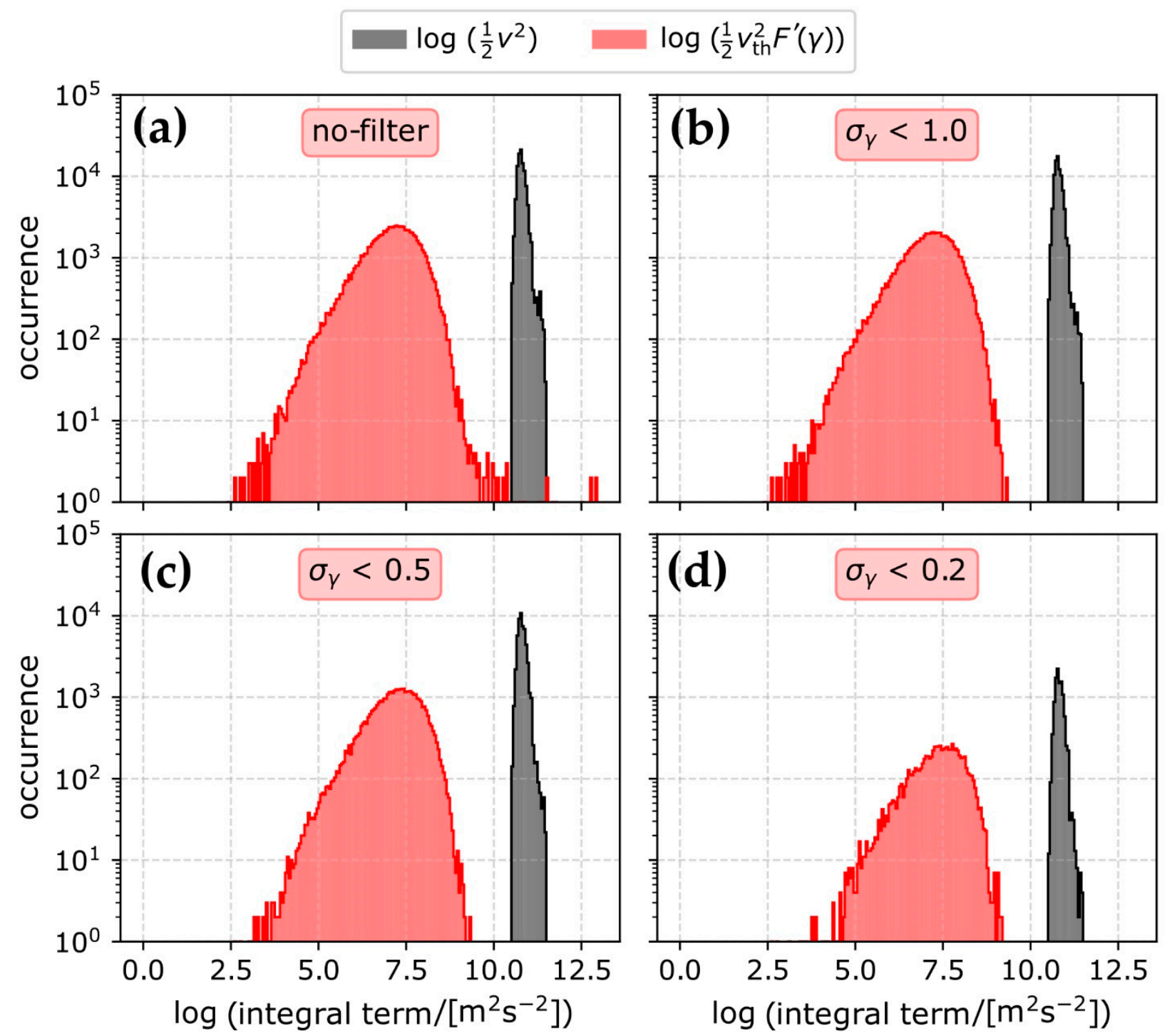

Figure 7. Histograms of (grey) $\frac{1}{2} v^{2}$ and (red) $\frac{1}{2} v_{\mathrm{th}}^{2} \gamma \ln _{\gamma}\left(\frac{\rho}{\rho_{*}}\right)$ terms of the complete Bernoulli integral for solar wind protons at 1 au observed by Wind during 1995. We present the cases for which we take into account (a) all $\sigma_{\gamma}$ values, (b) only data with $\sigma_{\gamma}<1$, (c) $\sigma_{\gamma}<0.5$, and (d) $\sigma_{\gamma}<0.2$.

\section{Discussion}

We examine the terms of the Bernoulli integral in solar wind protons at $\sim 1$ au. First, we show that $\frac{1}{2} v^{2}$ is typically by $\sim 2$ orders of magnitude larger than $\frac{1}{2} v_{\text {th }}^{2}$ and $\frac{1}{2} v_{\mathrm{A}}^{2}$. We then verify that the second term $\frac{1}{2} v_{\text {th }}^{2}\left(\frac{\gamma}{\gamma-1}\right)$ of the integral for non-isothermal plasmas, may become significant for $\left|\frac{\gamma}{\gamma-1}\right|>5$, which corresponds to $\frac{5}{6}<\gamma<\frac{5}{4}$. Moreover, we quantify the relative standard deviation of the Bernoulli integral within short intervals, assuming 
different $\gamma$ values. Our results show that the second and third terms of the integral affect the overall deviation of the integral by just a few percent, even for $\gamma \sim 5 / 6$ and $\gamma \sim 5 / 4$.

We further quantify the error of the second term as a function of the polytropic index uncertainty. We show that for the range of $\gamma$ values for which the term becomes significant $\left(\left|\frac{\gamma}{\gamma-1}\right|>5\right)$, the absolute value of its relative uncertainty exceeds $50 \%$, even for small $\gamma$ uncertainties (e.g., $\frac{\delta_{\gamma}}{\gamma}=0.1$ ). Therefore, in order to maintain a high accuracy in the calculations of the Bernoulli integral, we need to ensure higher accuracy of $\gamma$ as $\gamma \rightarrow 1$.

Based on our results, we could potentially drop the less significant terms of the Bernoulli integral without reducing the accuracy of the calculations. In other words, we could ignore the second and the third terms of the integral, or just the second term for a reasonable range of $\gamma$ close to unity. Note that by excluding the second term, we also reduce the large uncertainties of the integral due to the uncertainty $\delta_{\gamma}$. Such an approach may be useful when accurate measurements of the plasma density, temperature, or magnetic field are not available.

The study by [5] derives $\gamma$ of solar wind protons at 1 au by applying linear fittings to $\ln (n)$ vs. $\ln (T)$ observations. They exclude all the intervals for which the linear correlation between $\ln (n)$ and $\ln (T)$ is smaller than a threshold value. The correlation filter excludes all cases with $\gamma \rightarrow 1$ (see Figure 3 of [5] and the extensive discussion in [21]). The authors simplify their calculations further, by neglecting the internal energy term and the magnetic term, exactly as in [4]. This simplification is equivalent to using the Bernoulli integral expression for incompressible, non-magnetized plasmas, which is independent of $\gamma$. Although [22] argue that this approach is not appropriate, we support that when analyzing solar wind protons at $1 \mathrm{au}$, there is no apparent impact on the results if the cases with $\gamma \rightarrow 1$ are properly treated or excluded from a large statistical sample.

We also investigate the complete Bernoulli integral, which is a continuous function of $\gamma$. We examine the significance of the thermal term against the dynamic term as calculated for the solar wind protons at $\sim 1$ au assuming $\gamma=5 / 6$ and $\gamma=5 / 4$, respectively. We show that the second term is even less significant in this consideration than it is when we use the non-isothermal expression in Equations (1) and (3). However, we need to emphasize that the significance of the second term can be very large for a range of $\gamma$ and $\frac{\rho}{\rho_{*}}$ values. For example, for $\frac{\rho}{\rho_{*}}=0.1$, the thermal term is $<-5 v_{\text {th }}^{2}$ as $\gamma \rightarrow 2$. For completeness, we repeat our calculations in the solar wind protons using $\gamma$ values determined from linear fittings to $\ln (n)$ vs. $\ln (T)$ data points within short subintervals. Our calculations verify that the second term of the integral is insignificant in solar wind protons at $\sim 1 \mathrm{au}$, especially when we exclude intervals with high uncertainty of the derived polytropic index.

\section{Conclusions}

This study examines the significance of the Bernoulli integral terms in solar wind protons at $\sim 1$ au. By examining the relevant theory and analyzing spacecraft data we conclude the following:

- For typical $\gamma$ values, the dynamic term $\frac{1}{2} v^{2}$ is the most significant term of the integral;

- The term that includes the plasma temperature (thermal speed) in the non-isothermal Bernoulli integral, becomes significant only for $\gamma \rightarrow 1$;

- When the non-isothermal expression is used, the relative uncertainty of the integral becomes significantly large as $\gamma \rightarrow 1$;

- On average, we can estimate very accurately the Bernoulli integral of solar wind proton plasma at $\sim 1$ au and its fluctuations in short intervals by excluding cases with $\gamma \sim 1$ or by using only the dynamic term;

- The term of the continuous Bernoulli integral that includes the thermal speed is also negligible for typical solar wind protons at 1 au. 
Author Contributions: Conceptualization, G.N. and G.L.; methodology, G.N. and G.L.; software, G.N.; validation, G.N., G.L. and M.I.D.; formal analysis, G.N.; investigation, G.N. and G.L.; resources, G.L. and M.I.D.; data curation, G.N.; writing—original draft preparation, G.N.; writing—review and editing, G.N., G.L. and M.I.D.; visualization, G.N.; supervision, G.N., G.L. and M.I.D.; project administration, G.N., G.L. and M.I.D.; funding acquisition, M.I.D. All authors have read and agreed to the published version of the manuscript.

Funding: This research was funded by NASA Grant 80NSSC21K0112.

Institutional Review Board Statement: Not applicable.

Informed Consent Statement: Not applicable.

Data Availability Statement: The data set we use in this study is publicly available at https:// cdaweb.gsfc.nasa.gov/index.html (accessed on 14 May 2021).

Conflicts of Interest: The authors declare no conflict of interest.

\section{References}

1. Verscharen, D.; Klein, K.G.; Maruca, B.A. The multi-scale nature of the solar wind. Living Rev. Sol. Phys. 2019, 16, 5. [CrossRef] [PubMed]

2. Parker, E.N. Interplanetary Dynamical Processes; Wiley-Interscience: Hoboken, NJ, USA, 1963.

3. Chandrasekhar, S. An Introduction to the Study of Stellar Structure; Dover Publications: New York, NY, USA, 1967.

4. Kartalev, M.; Dryer, M.; Grigorov, K.; Stoimenova, E. Solar wind polytropic index estimates based on single spacecraft plasma and interplanetary field measurements. J. Geophys. Res. 2006, 111, A10107. [CrossRef]

5. Nicolaou, G.; Livadiotis, G.; Moussas, X. Long-term variability of the polytropic index of solar wind protons at 1 AU. Sol. Phys. 2014, 289, 1371-1378. [CrossRef]

6. Pang, X.; Cao, J.; Liu, W.; Ma, Y.; Lu, H.; Yang, J.; Li, L.; Liu, X.; Wang, J.; Wang, T.; et al. Polytropic index of central plasma sheet ions based on MHD Bernoulli integral. J. Geophys. Res. 2015, 120, 4736-4747. [CrossRef]

7. Livadiotis, G. Using kappa distributions to identify the potential energy. J. Geophys. Res. 2018, 123, 1050-1060. [CrossRef]

8. Livadiotis, G. Long-term independence of solar wind polytropic index on plasma flow speed. Entropy 2018, 20, 799. [CrossRef] [PubMed]

9. Nicolaou, G.; Livadiotis, G. Long-term correlations of polytropic indices with kappa distributions in solar wind plasma near 1 au. Astrophys. J. 2019, 884, 52. [CrossRef]

10. Park, J.-S.; Shue, J.-H.; Nariyuki, Y.; Kartalev, M. Dependence of Thermodynamic Processes on Upstream Interplanetary Magnetic Field Conditions for Magnetosheath Ions. J. Geophys. Res. 2019, 124, 1866-1882. [CrossRef]

11. Livadiotis, G. Turbulent Heating in Solar Wind Thermodynamics. Astrophys. J. 2019, 887, 117. [CrossRef]

12. Dayeh, M.A.; McComas, D.J.; Allegrini, F.; De Majistre, B.; Desai, M.I.; Funsten, H.O.; Janzen, P.; Livadiotis, G.; Randol, B.M.; Reisenfeld, D.B.; et al. Effects of Fast and Slow Solar Wind on the Energetic Neutral Atom (ENA) Spectra Measured by the Interstellar Boundary Explorer (IBEX) at the Heliospheric Poles. Astrophys. J. 2012, 749, 50. [CrossRef]

13. Kasper, J. Solar Wind Plasma: Kinetic Properties and Micro-Instabilities. Ph.D. Thesis, Massachusetts Institute of Technology, Cambridge, MA, USA, 2003.

14. Nicolaou, G.; Livadiotis, G.; Wicks, R.T.; Verscharen, D.; Maruca, B. Polytropic Behavior of Solar Wind Protons Observed by Parker Solar Probe. Astrophys. J. 2020, 901, 26. [CrossRef]

15. Livadiotis, G.; Nicolaou, G. Relationship between polytropic index and temperature anisotropy in space plasmas. Astrophys. J. 2021, 909, 127. [CrossRef]

16. Livadiotis, G. Superposition of polytropic in the inner heliosheath. Astrophys. J. Suppl. Ser. 2016, 223, 1-13. [CrossRef]

17. Silva, R., Jr.; Plastino, A.R.; Lima, J.A.S. A Maxwellian path to the q-nonextensive velocity distribution function. Phys. Lett. A 1998, 249, 401-408. [CrossRef]

18. Yamano, T. Some properties of q-logarithm and q-exponential functions in Tsallis statistics. Phys. A Stat. Mech. Appl. 2002, 305, 486-496. [CrossRef]

19. Livadiotis, G.; McComas, D.J. Beyond kappa distributions: Exploiting Tsallis statistical mechanics in space plasmas. J. Geophys. Res. 2009, 114, A11105. [CrossRef]

20. Livadiotis, G.; Desai, M.I.; Wilson, L.B., III. Generation of kappa distributions in Solar Wind at 1 au. Astrophys. J. 2018, 853, 142. [CrossRef]

21. Nicolaou, G.; Livadiotis, G.; Wicks, R.T. On the Calculation of the Effective Polytropic Index in Space Plasmas. Entropy 2019, 21, 997. [CrossRef]

22. Pang, X.; Wang, F.; Ceng, X.; Wang, X.; Deng, Z.; Zhang, Q.; Duan, P.; Xu, L. Polytropic Index of the Solar Wind Ions Near the Earth Calculated Using a Homogeneous Magnetohydrodynamic Bernoulli Integral. Astrophys. J. 2020, 904, 167. [CrossRef] 